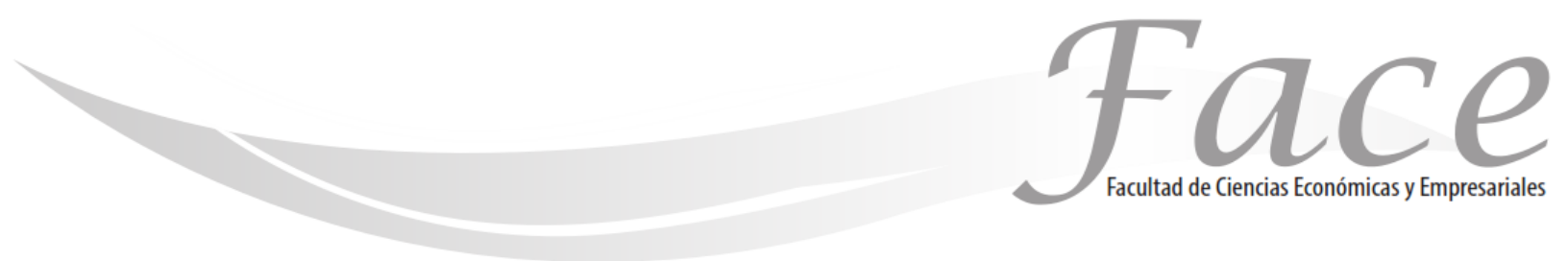

ISSN Impreso: 1794-9920

ISSN Electrónico: 2500-9338

Volumen $18-\mathrm{N}^{\circ} 1$

Año 2018

Págs. 6 - 21

\title{
REFLEXIONES EN LA APLICACIÓN DEL MONOTRIBUTO E IMPUESTO SOBRE LA RENTA Y COMPLEMENTARIO EN LOS COMERCIANTES DE MENORES INGRESOS EN COLOMBIA
}

Fecha de Recepción: 2 de Diciembre 2017

Fecha de Aprobación: 20 de Mayo 2018

\author{
Deivi David Fuentes Doria * \\ Enlace ORCID: https://orcid.org/0000-0002-0699-286X \\ Kevin Díaz Sánchez ** \\ Enlace ORCID: https://orcid.org/0000-0003-3557-4357 \\ Jimmy Ferney Urueña Majul *** \\ Enlace ORCID: https://orcid.org/0000-0002-6721-8368 \\ Johana Elisa Fajardo Pereira ${ }^{* \star * \star *}$ \\ Enlace ORCID https://orcid.org/0000-0001-7963-9349
}

\section{Resumen:}

El objetivo del material de estudio fue explicar de manera detallada lo relacionado al impuesto denominado monotributo, creado con el artículo 165 de la ley 1819 del 2016 que incluyo el artículo 903 del estatuto tributario nacional, haciendo un comparativo de la liquidación de comerciante mediante la opción monotributista y las rentas cedulares no laborales. La metodología utiliza en el estudio fue de tipo documental, teniendo en cuenta la nueva reforma estructural y decreto reglamentarios 978 de mayo del 2017 y 975 de junio del 2017. En este sentido el gobierno nacional adopta la alternativa opcional para aquello comerciante de menores ingresos con el fin de reducir la carga formales y sustanciales e impulsar la formalidad y general facilitar el cumplimiento de las obligaciones fiscales de los contribuyentes que voluntariamente se acoja al tributo. Se busca explicar a los lectores los beneficios que trae consigo la adopción voluntaria como también una descripción practica para realizar los cálculos establecido en el artículo 908 del estatuto tributario en comparación con la liquidación de impuesto de renta del articulo 341 ET. Los resultados muestran que es menos oneroso la aplicación de las rentas cedulares que la adopción del monotributista.

\section{Palabras Clave:}

* Contador Público, Doctor en Ciencia Mención Gerencia de la Universidad Rafael Belloso Chacín. Actualmente desempeña el cargo de docente tiempo completo y coordinador de investigación del programa de contaduría Pública sede Montería, vinculado el grupo de investigación GIPE. Correo Electrónico: deivi.fuentesd@campusucc.edu.co

**. Estudiante de pregrado en contaduría pública. Correo electrónico: kevin.diaz@campusucc.edu.co

*** Estudiante de pregrado en contaduría pública. Correo Electrónico: iimmy.uruena@campusucc.edu.co

**** Contador público, Especialista en gerencia de impuesto universidad Cooperativa de Colombia, Maestrante en Gerencia empresarial, Universidad Rafael Belloso Chacín. Correo Electrónico: Johana.fajardop@campusucc.edu.co 


\title{
FINANCIAL PERFORMANCE OF THE LARGE COMPANIES OF THE LEATHER, SHOE AND LEATHER SECTOR IN COLOMBIA
}

\begin{abstract}
:
The purpose of the study material was to explain in detail what is related to the tax denominated monotributo, created with article 165 of law 1819 of 2016 that included article 903 of the national tax statute, making a comparison of the merchant settlement through the option monotributista and non-labor slate income. The methodology used in the study was documentary, taking into account the new structural reform and regulatory decree 978 of May 2017 and 975 of June 2017. In this sense, the national government adopts the optional alternative for those merchants of lower income with in order to reduce the formal and substantial burden and promote formality and general facilitate compliance with the tax obligations of taxpayers who voluntarily accept the tax. It seeks to explain to readers the benefits of voluntary adoption as well as a practical description to perform the calculations established in article 908 of the tax statute in comparison with the income tax settlement of article $341 \mathrm{ET}$. The results show that the application of the slate income is less expensive than the adoption of the monotributista.
\end{abstract}

Keywords: Monotributo, income tax, non-labor schedular income.

\section{REFLEXÕES SOBRE A APLICAÇÃO DA MONOTRIBUTO, ATO DE IMPOSTO DE RENDA E IMPOSTOS COMPLEMENTARES AOS PEQUENOS FORNECEDORES NA COLÔMBIA}

\section{Resumo:}

O objetivo do projeto de produção é o mesmo, editorial de sua versão do teste 1819 de 2016 denominado monotributo, creado con el artículo 165 de la ley 1819 del 2016 que é incluido no artigo 903 do tributo nacional, haciendo un comparativo de la liquidación de comerciante mediante a opción monotributista y las rentas cedulares no laborales. A metodologia pode ser usada no estudio fue de tipo documental, tenando-se a nueva reforma estructural e decreto reglamentarios 978 de mayo del 2017 y 975 de junio del 2017. Este é o sentido nacional adoptado a alternativa opcional para aquilar o comerciante de menores ingresos con O Financiamento da Carga Reduzida e Suporta as Garantias e os Impulsionadores da Governança Financeira e Social, em geral, o cumprimento das obrigações fiscais das contribuições que voluntariamente se aplicam ao tributo. Se busca explicar os benefícios que realizam a tomada de decisão voluntária, como também fazer uma descrição para realizar os cálculos do artigo 908 do estatuto tributário em comparação com a liquidação de imposto de renda da articulação $341 \mathrm{ET}$. Los resultados muestran que es menos oneroso a aplicação das rentas cedulares que a adopção do monotributista.

Palavras chave: Monotributo, imposto de renda, receita horária não trabalhista. 
ISSN: 1794-9920 - ISSN Electrónico: 2500-9338

Enero - Julio de 2018

Volumen 18 Número 1, Año 2018 Págs. 6 - 21

\section{INTRODUCCIÓN:}

En América Latina la constante informalidad del trabajo, es una de las principales dificultades que se presenta en lucha de la desigualdad, esta tiene un impacto en la tributación y el mercado de trabajo, generando en las regiones los peores indicadores de desigualdad que se ven acompañados por niveles de carga tributaria reducidos. Situación que se combina con un alto porcentaje de trabajadores que se desempeñan en trabajos no registrados, produciendo alto niveles de riesgo asociados a enfermedades profesionales.

Las cifras presentadas por la Organización Internacional del Trabajo (OIT, 2014) se estimaba que por lo menos 130 millones de latinoamericanos viven de la informalidad, esto significa vivir con inestabilidad laboral, bajos ingresos, malas condiciones laborales, desprotección del estado, entre otras, la mayoría de las personas no se incorporan a la economía informal por elección, sino por la necesidad de sobrevivir.

Algunos países de la región hace décadas han implementado regímenes simplificados de tributación para pequeños y micro empresarios con el fin de conseguir su incorporación a los registros tributarios; en 1997 Brasil comenzó a aplicar el "SIMPLES", este incorporaba en un solo pago, la sustitución de varios impuestos y recursos de la seguridad social. En 1998 Argentina, siguió esta tendencia, aunque con diferente técnica presuntiva, y con diferentes recursos de la seguridad social sustituidos, posteriormente en 2001 Uruguay decide combinar estos esquemas con las contribuciones para el financiamiento de la seguridad social.

En tales diseños y tratamientos especiales, se busca gravar a las personas naturales pertenecientes a los pequeños y micro contribuyentes, excluyéndolos en cierta medida de las normas generales del impuesto sobre la renta, estableciendo métodos alternos de determinación del impuesto e imponiendo tasas más reducidas. Método que ha permitido a los países de América Latina "descomprimir" la carga de trabajo de las administraciones de impuestos y así de esta manera mejorar sus capacidades de fiscalización.

Al respecto, González (2006) señala que la mayoría de los países de la región la instrumentación de regímenes especiales de tributación para los pequeños contribuyentes no ha tenido como objeto primordial la recaudación tributaria, sino resolver una problemática de administración

tributaria con la finalidad de incorporar a la formalidad a un amplio sector de contribuyentes de escasa significación económica.

Asimismo, el autor menciona que, en la actualidad, en los países de América Latina se aplican diversos regímenes tributarios a los pequeños y micro contribuyentes, con diferentes técnicas presuntivas, sustituyendo desde un solo impuesto a varios y en algunos supuestos también a los recursos de la seguridad social, con la característica que en muchos países existe más de un régimen especial de tributación.

Al respecto de la implementación de estos regímenes en la mayoría de los países latinoamericanos se busca facilitar el recaudo de impuestos de los pequeños y medianos contribuyentes de baja significación fiscal, los cuales constituyen el sector más difícil de controlar y que en general no cumplen con su obligación tributaria, estos responden a ciertas características como son los bajos ingresos, condición de informalidad, bajo nivel educativo, poco conocimiento de sus deberes tributarios, entre otros. De esta manera, se busca la simplificación de las normas legales y trámites administrativos, apuntando con ello a brindarles un mecanismo para atraer a la formalidad a los agentes económicos, asimismo, mejorar el manejo de los recursos de la administración tributaria.

En lo que se refiere a Colombia, a partir de la ley 1819 del 29 de diciembre de 2016, esta realiza diferentes cambios en el ET vigente, dentro de los cuales se encuentra la creación del Monotributo en el artículo 904 de la ley en mención, este es un impuesto de carácter opcional de determinación integral, de causación anual, que sustituye el impuesto sobre la renta y complementarios a cargo de los contribuyentes que opten voluntariamente por acogerse al mismo, tributo está dirigido a personas naturales que cumplan con los requisitos establecidos en el artículo 905 de la ley expuesta. 
Deivi David Fuentes Doria - Kevin Díaz Sánchez - Jimmy Ferney Urueña Majul - Johana Elisa Fajardo Pereira superiores a 1.400 UVT e inferiores 3.500 UVT, en valores

La razón principal de dicho impuesto es reducir la informalidad, facilitar el cumplimiento de la obligación tributaria, evitar la evasión de impuestos y simplificar el recaudo de impuestos para los comerciantes de bajos ingresos.

Aunque existe un valor agregado asociado al monotributo, relacionado al emprendimiento de los comerciantes, dado que el emprendimiento permite a los comerciantes mejorar sus condiciones informales. Abriendo nuevos mercados de beneficios, asociados a crédito, incentivos gubernamentales e incluso riquezas y crecimiento competitivo asociado al desarrollo tecnológico y humano. (Arias y Pérez 2015)

Ahora bien, si se relaciona la adopción del monotributo con la productividad y crecimiento de los comerciantes, (Rincon de Parra 2001) los incrementos de productividad no solo no conducen definitivamente hacia la mejora de calidad; sino que, incluso, la pueden deteriorar. Se propone entonces a la luz de la experiencia, que se dé prioridad a las estrategias de garantía de calidad, las cuales, en consecuencia, incrementan la productividad y minimizan los costos, y no a las inversas.

Por otro lado, en Colombia, existe un alto porcentaje de comerciantes que se dedican al comercio al por menor, según cifras emitidas por el Departamento Administrativo Nacional de Estadística (DIAN 2018) boletín técnico del 09 de marzo, informó que para el trimestre comprendido entre noviembre de 2017 y enero del 2018 la cifra de ocupados informales era del 48,1\%, por esta razón en Colombia estas personas se encuentran a la expectativa de este nuevo impuesto. Tal es el desconocimiento que a corte del 31 de julio del año 2017, según las cifras expresadas por la Dirección de Impuestos y Aduanas Nacionales-DIAN, en el Registro Único Tributario (RUT), solo 59 ciudadanos habían optado por inscribirse a este nuevo régimen tributario: 35 realizaron su inscripción como contribuyentes de Monotributo BEPS y 24 como contribuyentes de Monotributo ARL. Por consiguiente, se hace necesario realizar el presente estudio, donde se pretende reflexionar aspectos del impacto adopción del Monotributo en los comerciantes.

En consideración a los beneficios el gobierno estableció los sujetos pasivos del tributo, estableciendo algunas condiciones en relación a los ingresos percibidos incluyendo los ordinarios y extraordinario iguales 0
2017 corresponde a la $\$ 44.602 .600$ y $\$ 111.506 .500$, rango que concuerda con las obligaciones formales de presentar declaración de renta según lo establecido el articulo 592 E.T y 499E.T para la declaración de impuesto sobre las ventas de régimen común. también podrán acogerse de manera voluntaria aquellos que hayan obtenido ingresos brutos ordinarios 0

extraordinarios inferiores a 1.400 UVT. Dichos ingresos no pueden provenir de rentas de trabajo y, si la persona obtuvo rentas de capital o dividendos, estos tampoco pueden superar el $5 \%$ del total de los ingresos de la persona.

Así mismo, de acuerdo con el artículo 905 del ET, podrán acogerse al sistema del monotributo solo las personas naturales (residentes o no residentes, pues la norma no hizo distinción) que cumplan al mismo tiempo las cuatro condiciones especiales que allí se mencionan, entre ellas las de realizar una o más de las actividades de comercio al por menor (aquellas que en el RUT empiezan con el código 47). Por tanto, siendo claro que en el monotributo se inscribirán personas naturales comerciantes, y que de acuerdo con los artículos 10 y 19 del Código de Comercio están obligados a inscribirse en el registro mercantil y llevar contabilidad, surge la inquietud acerca de si la DIAN les exigirá o no exhibir dicha contabilidad al momento de auditar su declaración anual del monotributo. En el pasado, mediante el Concepto 15456 de 2006 (aún vigente), la DIAN dispuso que a las personas naturales comerciantes y responsables del IVA en el régimen simplificado, no les exigiría contabilidad, pero sí el libro fiscal de operaciones diarias (ver artículo 616 del ET que aún sigue vigente y el concepto DIAN 46572 de diciembre de 1999 donde se confirma que no se pueden aplicar sanciones a quienes no lleven el libro de operaciones diarias o lo lleven atrasado). Sin embargo, aunque ese concepto exonere de llevar contabilidad para efectos fiscales a los responsables del régimen simplificado del IVA, se entiende que en el monotributo también estarán inscritas personas que no son responsables del IVA (por ejemplo, quienes comercian solo con bienes excluidos o exentos.

De tal modo que el articulo busca conocer los requerimientos que debe tener las personas naturales que adopten por monotributo, las responsabilidades formales y sustanciales, los beneficios asociados a la adopción del 
ISSN: 1794-9920 - ISSN Electrónico: 2500-9338

Enero - Julio de 2018

Volumen 18 Número 1, Año 2018 Págs. 6 - 21

tributo, haciendo un comparativo del valor a pagar siendo régimen del impuesto sobre la renta y monotributista.

\section{MARCO REFERENCIAL:}

\subsection{Monotributo}

El Monotributo es un Sistema de Régimen Simplificado que se concentra en un único tributo, de acuerdo (Sánchez, Rodríguez y Zumaeta, 2000). el monotributo busca como objetivo generar alternativa de dinamizar los impuesto y responsabilidades sustanciales de los pequeños comerciantes y productores, el cual les permite de forma mensual realizar el pago correspondiente a sus impuestos a través de una cuota mensual fijada en función a sus compras y/o ingresos, con la que se sustituye el pago de las diversas cargas tributarias.

Asimismo, (Calero, 2012), define el monotributo como una alternativa que simplifica el cumplimiento de obligaciones impositivas generadas de pequeños comerciantes buscado una incorporación de las personas naturales independiente a la formalización social

Adicionalmente el autor Bustamante (2013), nos indica que el Monotributo no debe ser concebido como una fuente de ampliación de recaudo fiscal a corto plazo, sino que este debe ser visto como aquella herramienta que estimulará la creación de empleo formal, así como la inclusión económica y social.

Del mismo modo David Moucharfiech (2011), nos proporciona un concepto acerca que el Monotributo, este tributo se deriva de la dificultad que se presenta al momento de llevar las obligaciones contables y tributarias, dado que no existe por parte de las administraciones fiscales un control, formal de los comerciantes

Por otro lado, el artículo 908 del E.T adicionado por la ley 1819 del 2016 establece una tabla para cálculo del valor a paga de acuerdo a la categoría establecida por el contribuyente en relación a los ingresos ordinarios y extraordinarios.
Tabla 1.

Rango del Monotributo

\begin{tabular}{lccc}
\hline & \multicolumn{1}{c}{ A } & B & C \\
\hline $\begin{array}{l}\text { Ingreso } \\
\text { brutos } \\
\text { Anuales }\end{array}$ & Minimo & 1400UVT2100 UVT & 2800 UVT \\
$\begin{array}{l}\text { Valor a pagar } \\
\begin{array}{l}\text { Componente } \\
\text { impuesto }\end{array}\end{array}$ & Maximo & 2100 UVT 2800 UVT & 3500 UVT \\
$\begin{array}{l}\text { Componente } \\
\text { aporte BEPS }\end{array}$ & 16 UVT & 24 UVT & 32 UVT \\
\hline
\end{tabular}

Fuente: Elaborado a partir del artículo 908 del estatuto tributario

Respecto al cambio de régimen, el artículo 915 del E.T establece que aquellos contribuyentes que vienen desarrollando su actividad como régimen común, pueden optar por el cambio al régimen monotributista siempre y cuando cumplan con todos los requisitos establecido durante un periodo anterior de 3 años.

Por otro lado los beneficio generado por la adopción del monotributo, se pierde Cuando dentro de los programas de fiscalización la Administración Tributaria establezca que el contribuyente no cumple los requisitos para pertenecer al monotributo, procederá a excluirlo del régimen, mediante resolución independiente en la cual se reclasificará al contribuyente en el régimen tributario que corresponda., así lo establece el artículo 913 E.T, asimismo el artículo 914 del mismo estatuto cuando el contribuyente incumpla con los pagos de acuerdo a la categoría establecida será objeto de exclusión u no podrá optar por un periodo de tres años.

En relación a la destinación de los componentes del monotributo el articulo 916 E.T establece que el recaudo del componente del impuesto nacional del monotributo se destinará a la financiación del aseguramiento en el marco del Sistema General de Seguridad Social, en Salud y en Riesgos Laborales. Para el primer caso, los recursos se presupuestarán en la sección del Ministerio de Salud y Protección Social, y serán transferidos a la Entidad Administradora de los Recursos de Sistema General de Seguridad Social en Salud. En el segundo caso, los recursos serán transferidos al Fondo de Riesgos Laborales. 


\subsubsection{Declaración y pago del monotributo}

Respecto a las declaraciones de pago, Castañeda (2003), que corresponde a las obligaciones que tienen los contribuyentes de cumplir con la obligación sustancial cuando exista a partir de los procedimientos fiscales un valor monetario a cancelar.

En tal sentido, los contribuyentes del monotributo deberán presentar una declaración anual en un formulario simplificado que para el efecto adopte la Dirección de Impuestos y Aduanas Nacionales, dentro de los plazos que señale el Gobierno Nacional. La declaración del monotributo deberá presentarse con pago en los bancos y demás entidades autorizadas para recaudar.

Por otro lado, el artículo 1.6.1.13.2.55. (DUT 738/2017). Establece que los componentes del monotributo BEPS se deberán pagar a partir del primer día hábil del mes de septiembre del año 2017 y hasta el 30 de enero de' 2018, Ios contribuyentes del monotributo BEPS podrán realizar abonos del componente de' impuesto de carácter nacional del monotributo BEPS, previamente a los componentes del monotributo riesgos laborales.

\subsubsection{Beneficios asociados al monotributo}

De acuerdo Vasco (2017) los beneficios inherente al monotributo corresponde al régimen de afiliación voluntaria de expansión de cobertura de servicios sociales, es decir aquellos contribuyentes de monotributo perteneciente a la categoría $C$ y que se encuentra afiliado al régimen subsidiado podrán aplicar la regla establecida en la ley 789 del 2002 relacionado a los beneficios de los trabajadores independiente correspondiente a los servicios de las cajas de compensación (actividades recreativas, capacitaciones), exceptuado el beneficio monetario que reciben los contribuyente de afiliaciones ordinaria.

Del mismo modo la adopción del monotributo permite a los comerciantes a genera valor organizativo a la entidad, a partir de la formalización inicia una etapa de crecimiento económico asociado a la toma decisiones de acuerdo a lo expresado por (Martinez A.G 2016) El individuo como tomador de decisiones se compone de una estructura cognitiva que le permite procesar información y crear conocimiento, así como también de estrategias que le ayuden a hacer frente a los problemas que constantemente enfrenta.

\subsection{Impuesto de renta y complementario}

El impuesto a las ganancias, en la regulación fiscal colombiana comprende básicamente al impuesto sobre la renta y su complementario de ganancias ocasional. El impuesto a las ganancias puede ser clasificado como corriente o diferido, según Vargas (2017) el impuesto sobre la renta corresponde al valor a pagar o recuperar por conceptos de impuestos a las ganancias y el cual se deriva de las rentas gravables del periodo, este impuesto llamado corriente correspondiente con el impuesto básico el cual se determina sobre la base gravable.

Ahora bien, el impuesto de renta y complementario se puede definir como un tributo que grava tanto a las personas naturales y jurídicas, entiendo como personas naturales todos los seres humanos cual se su sexo y edad referente por al código civil en el artículo 74 y personas jurídicas como aquellos entes constituido a través de una sociedad comercial cual sea su objeto social, como lo mencionado en los artículos 12 y 13 del estatuto tributario. En este sentido según la clasificación de su origen de impuesto, según lo determinado en la ley, corresponde a un impuesto de carácter permanente, de manera directa que gravas los ingresos percibido en un periodo fiscal, Según su ordenamiento territorial es aplicado en todo el territorio colombiano, es por eso que es denominado de orden nacional y por consiguiente administrado por las DIAN como sujeto activo

\subsubsection{Sujeto Pasivo de impuesto de renta y complementario}

Los sujetos pasivos según el concepto unificado anterior, corresponde al deudor de la obligación tributaria, es decir el causante del hecho generados del impuesto, que para el caso del impuesto sobre la renta es la obtención de ingresos susceptible de producir un aumento en el patrimonio, por otro lado los sujetos pasivos pueden clasificarse de acuerdo a lo establecido por Escobar G.C (2005) en sujetos pasivos de Derecho, quienes son responsables de actuar como recaudadores y deben cumplir con la obligación que le impone el estado para no ser sancionados por la administración, por otro lado los 
ISSN: 1794-9920 - ISSN Electrónico: 2500-9338

Enero - Julio de 2018

Volumen 18 Número 1, Año 2018 Págs. 6 - 21

sujetos pasivos económico es la persona que adquiere bienes o servicios quien soporta o asume el impuesto, siendo parte de la obligación tributaria.

De mismo modo es importante destacar que las personas naturales en Colombia son sujetas al impuesto de acuerdo algunos requisitos establecido en los artículos 592-594 del estatuto que relaciona los ingresos, patrimonio, consignaciones y movimiento, es así que la norma establece que los contribuyentes que no declaran donde incluye los contribuyentes personas naturales y sucesiones ilíquidas que no sean responsables del impuesto a las ventas, que en el respectivo año o período gravable hayan obtenido ingresos brutos inferiores a 1.400 UVT y que el patrimonio bruto en el último día del año o período gravable no exceda de 4.500 UVT, del mismo modo Sin perjuicio de lo dispuesto en el artículo 592, para no estar obligado a presentar declaración de renta y complementarios se tendrán en cuenta los consumos mediante tarjeta de crédito durante el año gravable no excedan la suma de1.400 UVT, el total de compras y consumos durante el año gravable no superen la suma de 1.400 UVT y el valor total acumulado de consignaciones bancarias, depósitos o inversiones financieras, durante el año gravable no exceda de 1.400 UVT.

Adicional a lo anterior las personas naturales 0 jurídicas, extranjeras, sin residencia o domicilio en el país, cuando la totalidad de sus ingresos hubieren estado sometidos a la retención en la fuente de que tratan los artículos 407 a 411, inclusive, y dicha retención en la fuente, así como la retención por remesas cuando fuere del caso, les hubiere sido practicada. Los contribuyentes señalados en el artículo 414-1 de este Estatuto y finalmente las personas naturales que pertenezcan al monotributo.

\subsubsection{Hecho Generador}

El hecho generado corresponde al presupuesto establecido en la ley cuya realización origina el nacimiento de la obligación sustancial, según lo definido por Corredor (2009) en materia tributaria el hecho generador es la manifestación eterna del hecho imponible, el cual es un hecho económico por la ley sustancial como elemento de la obligación tributaria. Asimismo, Giannini, A. (1957). Menciona que el hecho generador se encuentra establecido en la legislación Colombia en el artículo 338 de
$\mathrm{CN}$, los cuales establece los elementos de la tributación en Colombia, que son necesarios y fundamentales para la creación de leyes, ordenanzas y acuerdo, por otro lado, el hecho generados haciendo referencia al impuesto sobre la renta y complementario se origina en la obtención de los ingresos ordinarios y extraordinario que generan un incremento patrimonial.

\subsubsection{Base gravable}

De acuerdo a lo establecido por Francos y Martínez (2010) la base del impuesto de rentas son todos los ingresos ordinarios y extraordinario obtenido por el ente económico, a partir de las disposiciones del articulo 26 ET establece la renta líquida gravable se determina así de la suma de todos los ingresos ordinarios y extraordinarios realizados en el año o período gravable, que sean susceptibles de producir un incremento neto del patrimonio en el momento de su percepción, y que no hayan sido expresamente exceptuados, se restan las devoluciones, rebajas y descuentos, con lo cual se obtienen los ingresos netos.

Asimismo, el autor destaca que los ingresos netos se restan, cuando sea el caso, los costos realizados imputables a tales ingresos, con lo cual se obtiene la renta bruta. De la renta bruta se restan las deducciones realizadas, con lo cual se obtiene la renta líquida. Salvo las excepciones legales, la renta líquida es renta gravable y a ella se aplican las tarifas señaladas en la ley.

\subsection{4 tarifa}

La tarifa corresponde al porcentaje o valor calculado a partir de la base gravable después de ser depurada con los costos, deducciones y rentas exentas establecida por la ley, desde el punto de punto del impuesto sobre la renta y su complementario de ganancias ocasionales (Monsalve, $R, 2005)$.

De acuerdo lo establecido en el artículo 241 ET que fue modificado por la ley 1819 2016, se establece dos tablas para cedulas laborales y pensiones, que tributan a través del numeral 1 del artículo 241 ET y las cedulas no laborales y de capital tributaran a través del numeral 2.

\subsubsection{Cedula No laboral}




\section{REFLEXIONES EN LA APLICACIÓN DEL MONOTRIBUTO E IMPUESTO SOBRE LA RENTA Y COMPLEMENTARIO EN LOS COMERCIANTES DE MENORES INGRESOS EN COLOMBIA}

Deivi David Fuentes Doria - Kevin Díaz Sánchez - Jimmy Ferney Urueña Majul - Johana Elisa Fajardo Pereira

De conformidad con las normas tributarias el artículo 107

La cedula no laboral, fue un concepto adoptado por el artículo 340 del estatuto tributario, estableciendo tal clasificación para aquellas personas naturales que no clasifique como rentas laborales y pensiones, puedan calcular su impuesto, sin importar el tipo de actividad, sea agrícola, pecuaria, minera, comerciales, intermediarios. Vasco R (2018).

En tal sentido las personas naturales en Colombia que desarrollen actividades mercantiles a partir del periodo 2017, debe aplicar los sistemas cedulados, cuando cumplen con las características establecida en el artículo 592 y 594 del estatuto tributario, obligados a declarar renta por ingresos laborales, patrimonio, consignaciones e inversiones.

Como toda renta líquida, los proceso para la obtención de la renta gravable, se establece de acuerdo al artículo 341 $\mathrm{ET}$, tomando el total de ingresos gravables, restando los ingresos que no constituye renta y ganancias ocasionales y costos y gastos procedentes imputables, siempre y cuando este soportados por el contribuyente. Seguidamente se debe limitar las exenciones y deducciones tomando el 10\% del resultado anterior descrito sin superar las 1000 uvt.

Como se puede notar la norma tributaria estableció limitaciones de deducciones y rentas exenta imputables a los contribuyentes, asociado de acuerdo al decreto 2250 del 2017, los pagos de gravamen a los movimientos financieros, intereses de vivienda, aportes a fondos voluntarios de pensiones, AFC

\subsubsection{Costos y Gastos}

De acuerdo a lo establecido por Pardo, N., Hoyos, J.I. y Monsalve, R. (2004). Los costos y gastos corresponden a erogaciones de recursos que destina las personas naturales 0 jurídicas para el desarrollo de su actividad comercial. Del mismo modo Rúa (2007) define los costos y gastos como todas aquellas erogaciones en que se incurre para obtención del ingreso y que cumplen con todos los requisitos y limitaciones para su procedencia de conformidad con los establecido en el estatuto tributario. del ET, establece acerca de los principios de causalidad, necesidad y proporcionalidad que deben cumplir los costos y gasto para ser deducible del cálculo de la renta líquida.

Finalmente, una vez el contribuyente obtenga la renta liquidad gravable debe calcula el impuesto teniendo en cuenta la tabla del artículo 241-2

Tabla 1.

Artículo 241-2

\begin{tabular}{|c|c|c|c|}
\hline \multicolumn{2}{|c|}{ Rangos UVT } & \multirow{2}{*}{$\begin{array}{c}\text { Tarifa } \\
\text { Marginal }\end{array}$} & \multirow[t]{2}{*}{ Impuesto } \\
\hline Desde & Hasta & & \\
\hline$>0$ & 600 & $10 \%$ & 10 \\
\hline$>600$ & 1000 & $10 \%$ & $\begin{array}{l}\text { (Base Gravable en UVT } \\
\text { menos } 600 \text { UVT) x 10\% }\end{array}$ \\
\hline$>1000$ & 2000 & $20 \%$ & $\begin{array}{l}\text { (Base Gravable en UVT } \\
\text { menos } 1000 \text { UVT) } x \\
20 \%+40 \text { UVT }\end{array}$ \\
\hline$>2000$ & 3000 & $30 \%$ & $\begin{array}{l}\text { (Base Gravable en UVT } \\
\text { menos } 2000 \text { UVT) } x \\
30 \%+240 \text { UVT }\end{array}$ \\
\hline$>3000$ & 4000 & $33 \%$ & $\begin{array}{l}\text { (Base Gravable en UVT } \\
\text { menos } 3000 \text { UVT) } x \\
33 \%+540 \text { UVT }\end{array}$ \\
\hline$>4000$ & $\begin{array}{l}\text { En } \\
\text { adelante }\end{array}$ & $35 \%$ & $\begin{array}{l}\text { (Base Gravable en UVT } \\
\text { menos } 4000 \text { UVT) } x \\
35 \%+870 \text { UVT }\end{array}$ \\
\hline
\end{tabular}

El contribuyente debe ubicarse de acuerdo a la renta líquida obtenida en la depuración del articulo 341 y calcula el valor a pagar en el rango determinado.

\section{METODOLOGÍA:}

El presente trabajo investigativo posee un enfoque mixto, investigaciones se fundamentan en un proceso inductivo como explorar y describir, y luego generar perspectivas teóricas, según lo expresado (Hernández, Fernández y Baptista, 2010).

Del mismo modo, teniendo en consideración el método como "la forma característica de investigar determinada por la intención sustantiva y el enfoque que la orienta" 
ISSN: 1794-9920 - ISSN Electrónico: 2500-9338

Enero - Julio de 2018

Volumen 18 Número 1, Año 2018 Págs. 6 - 21

(Rodríguez, Gil y García, 1996, P.40), en el presente estudio se utiliza el método fenomenológico. La investigación fenomenológica es la descripción de los significados vividos, existenciales. La fenomenología procura explicar los significados en los que estamos inmersos en nuestra vida cotidiana, y no las relaciones estadísticas a partir de una serie de variables, el predominio de tales o cuales opiniones sociales, o la frecuencia de algunos comportamientos. (Rodríguez, Gil y García, 1996, P.40).

El diseño de la investigación sitúa en no experimental, dado que el investigador no hace manipulación de las categorías de estudio. En este sentido, la investigación de tipo permite abordar de manera detallada análisis documentales, textos, normas tributarias.

La investigación se ubica dentro de los estudios documentales, dado que se hizo uso de leyes tributaria, decretos, resoluciones, conceptos tributario

\section{RESULTADOS:}

El presente apartado corresponde a la depuración del impuesto de dos comerciantes que desean adoptar el monotributo como opción de formalización, del mismo modo, se realiza el cálculo del impuesto sobre la renta ordinario no laboral y monotributo, determinado cual genera mayores beneficios sustanciales al contribuyente.

Caso 1. Persona natural, dedicado a la comercialización de equipos electrónicos, percibe durante el año 2017 ingresos brutos totales de $\$ 80.000 .000$, costo y gastos asociados debidamente soportados con facturar $\$ 53.000 .000$ de mercancía y servicios públicos. Posee gastos de GMF 4 xmil por transacciones asociadas a la actividad por valor $\$$ 200.000. patrimonio bruto de acuerdo a propiedades y saldo en cuentas bancarias $\$ 90.000 .000$.

Calculo de tributo modalidad Monotributo

El primer paso para contribuyente objeto de liquidación del monotributo es determinar la categoría que pertenece de acuerdo a la tabla del artículo 908 del E.T, la cual se obtiene dividiendo los ingresos brutos entre el valor de la UVT del periodo a liquidar, es decir:

$\$ 80.000 .000 / 31859=2,511.06$
Como se puede notar se toma la UVT del periodo 2017 la cual corresponde a 31.859 , seguidamente se ubica el valor calculado en el rango de la tabla

Tabla 2.

Calculo de Impuesto a Pagar

\begin{tabular}{lcc}
\hline & \multicolumn{1}{c}{ B } \\
\hline $\begin{array}{l}\text { Ingreso brutos } \\
\text { Anuales }\end{array}$ & Minimo & 2100 UVT \\
Valor a pagar & Maximo & 2800 UVT \\
$\begin{array}{l}\text { Componente } \\
\text { impuesto }\end{array}$ & 24 UVT \\
$\begin{array}{l}\text { Componente } \\
\text { aporte BEPS }\end{array}$ & 19 UVT \\
\hline
\end{tabular}

Fuente: Autor

Como se puede notar el contribuyente de acuerdo a los ingresos pertenecerá a la

categoría B, cancelando un valor de 24 UVT divididos 19UVT componente de impuesto y 5 UVT componente BEPS.

Valor a pagar $=24$ UVT $* 31859=764.616$

De allí se establece de acuerdo al procedimiento el componente:

Impuesto $=19 U V T^{*} 31.859=605.321$

BEPS $=5$ UVT $^{*} 31859=159.295$

Como se puede notar en el cálculo del impuesto por la modalidad monotributista, no tiene en cuenta costos y gastos asociado a la actividad, asimismo las rentas exentas y deducciones no son depuradas de los ingresos. Facilidad que permite a cualquier contribuyente realiza un simple procedimiento aritmético determinado la categoría y pagando el valor correspondiente de la tabla.

Respecto al cálculo anterior el monotributista debe pagar a través del formato 250 que fue expedido por la resolución 00002 del 15 enero 2018 presentado de forma virtual. Aquí se deja ver que todos lo contribuyente que adopte la alternativa monotributo, debe diligencia su firma digital para el cumplimiento de presentación de la declaración por los medios digitales. 
Cuando la declaración del monotributo se presente de manera extemporánea, la sanción por cada mes o fracción de mes calendario de retardo será equivalente al tres por ciento $(3 \%)$ del total del impuesto a cargo, sin exceder del ciento por ciento (100\%).

Tabla 3.

\section{DEPURACIÓN DE LA CEDULA POR RENTAS NO LABORALES}

(Art. 341 E.T. Ley 1819 / 2016 Art. 1)

TOTAL INGRESOS BRUTOS POR RENTAS NO LABORALES INGRESOS POR ACTIVIDAD ORDINARIA

MENOS: INGRESOS NO CONSTITUTIVOS DE RENTA

MENOS: COSTOS Y GASTOS

Costos de venta de productos

SUBTOTAL 1.

MENOS: DEDUCCIONES

a. Dependientes económicos

Límite 1: $10 \%$ de Ingresos $(\$ 80.000 .000 \times 10 \%)$

b. G.M.F. Certificado

$(200.000 \times 50 \%)$

TOTAL DEDUCCIONES

MENOS: RENTAS EXENTAS

Depuración de la base para el $10 \%$

Total ingresos por rentas no laborales

Menos: INCR

Menos: Costos y Gastos

Menos: Deducciones

Menos: Rentas exentas

SUBTOTAL BASE PARA EL $10 \%$

$\$ 27.000 .000 \times 10 \%$

RENTA LIQUIDA GRAVABLE POR RENTAS NO LABORALES FINAL

Según tabla Art. 241 E.T. Núm. 1 - UV

$\$ 26.900 .000 / 31.859=844.345$ UVT

$(>0-1090$ UVT $)=0 \%$

TOTAL IMPUESTO A CARGO

\begin{tabular}{|c|c|c|}
\hline RALES & $\$ 80.000 .000$ & $\begin{array}{l}\$ 80.000 .000 \\
\$ 0\end{array}$ \\
\hline No aplica & $\begin{array}{l}\$ 53.000 .000 \\
\text { So }\end{array}$ & $\begin{array}{l}\$ 53.000 .000 \\
\underline{\$ 27.000 .000} \\
\underline{100.000} \\
\underline{\$ 0}\end{array}$ \\
\hline$\$ 50.000$ & $\begin{array}{r}\$ 100.000 \\
\$ 0\end{array}$ & \\
\hline $\begin{array}{l}\$ 80.000 .000 \\
\$ 0 \\
\$ 53.000 .000 \\
\$ 100.000 \\
\$ 0 \\
\$ 27.000 .000 \\
\$ 2.700 .000\end{array}$ & & $\$$ \\
\hline$\$ 26.900 .000$ & & $\$ 26.900 .000$ \\
\hline
\end{tabular}

Como se puede apreciar en el cálculo del impuesto adoptando por la cedula no laboral el contribuyente deberá cancelar un impuesto correspondiente $\$ 0$, aplicando todas las normas establecida en el estatuto y cumplimiento con la obligación formal de presentar el formato 210 cedulado para personas naturales. A diferencia de los contribuyentes monotributista el contribuyente cedulado puede presentar su declaración de manera litográfica ante una entidad financiera, sin la necesidad de solicitar firma digital. 
ISSN: 1794-9920 - ISSN Electrónico: 2500-9338

Enero - Julio de 2018

Volumen 18 Número 1, Año 2018 Págs. 6 - 21

En comparación del contribuyente se puede expresar que generar mayores beneficios adoptar los sistemas cedulados dado que el impuesto a pagar por la alternativa en $\$ 0$, al contrario de la opción monotributista que el valor a canelar $\$ 764.616$. En ambos casos el contribuyente cumple con su obligación tributaria antes los entes fiscales.

Ahora bien, es preciso analizar otro caso de contribuyente que clasifique como categoría $A$, para mostrar el comportamiento del impuesto

\section{Caso 2}

Persona natural comerciante muebles para hogar, recibió durante el 2017 ingreso bruto total de \$ 102.000.000, costos y gastos asociado a la actividad $\$ 80.000 .000$, GMF de $\$ 500.000$, patrimonio de $\$ 100.000 .000$, deudas de $\$$ 50.000 .000 .

Como vimos anteriormente, es importante determinar si el comerciante es sujeto del monotributo, por los ingresos menores de $\$ 111.506 .500$, establecimiento menor de $50 \mathrm{~m} 2$.

\section{$\$ 102.000 .000 / 31859=3.201 .60$ UVT}

Como se puede notar se toma la UVT del periodo 2017 la cual corresponde a 31.859 , seguidamente se ubica el valor calculado en el rango de la tabla

Tabla 4.

Calculo de impuesto a pagar

\begin{tabular}{llc}
\hline \hline & & 2800 \\
Ingresos brutos & Minimo & \\
anuales & Maximo & 3500 \\
Valor a pagar & & 32 UVT \\
Componete & & 26 UVT \\
Impuesto & & 6 UVT \\
\hline Aporte BEPS & & \\
\hline
\end{tabular}

Fuente: Autor
Como se puede notar el contribuyente de acuerdo a los ingresos pertenecerá a la categoría $\mathrm{C}$, cancelando un valor de 32 UVT divididos 26UVT componente de impuesto y 6 UVT componente BEPS.

Valor a pagar $=32$ UVT * $31859=1.019 .488$

De allí se establece de acuerdo al procedimiento el componente:

Impuesto $=26 \mathrm{UVT}^{\star} 31.859=828.334$

BEPS $=6$ UVT $^{*} 31859=191.154$

Como se puede apreciar en el cálculo realizado el contribuyente si adopta el mecanismo de monotributo, deberá liquidar un impuesto correspondiente a la categoría C de 32 UVT, por valor de $\$ 1.019 .488$, que finalmente quedaría distribuida en los dos componentes para BEPS \$ 191.154 e impuesto $\$ 828.334$.

AL igual que el caso anterior los valores correspondientes a costos y gastos asociado del periodo no influyen en el cálculo de impuesto, llegando a pensar en aquellos comerciantes que tengan perdidas en un periodo fiscal por circunstancias del mercado, deberán pagar impuesto, dado que los costos y gastos asociado del periodo no hacen parte del cálculo de la depuración de la base liquida del monotributo, es allí donde los comerciantes deben empezar analizar una adecuada planeación financiera y tributaria, de acuerdo lo expresado por (Hernández et al. 2013) En Colombia los administradores y propietarios de las Mipymes presentan un desconocimiento generalizado sobre la importancia de la planeación financiera para gestionar el desarrollo, crecimiento y fortalecimiento de este tipo de organizaciones. Se hace necesario que las Mipymes, realicen un análisis de los estados financieros apoyado en un diagnóstico financiero, para observar la situación de la empresa frente a la fiscalidad. 

favorable la aplicación del sistema cedulado, dado que su cálculo de acuerdo al artículo $341 \mathrm{ET}$, el impuesto a pagar por el periodo 2017 corresponde \$195.000. Al contrario de la aplicación de monotributo, lo cual generaba para el contribuyente el saldo a tributar de $\$ 1.019 .488$. Valor que comparado con el de renta cedular supera un $80 \%$ del valor tributado. Factor que debe tener en cuenta los comerciantes que quiera adoptar la aplicación del monotributo.

Tabla 5.

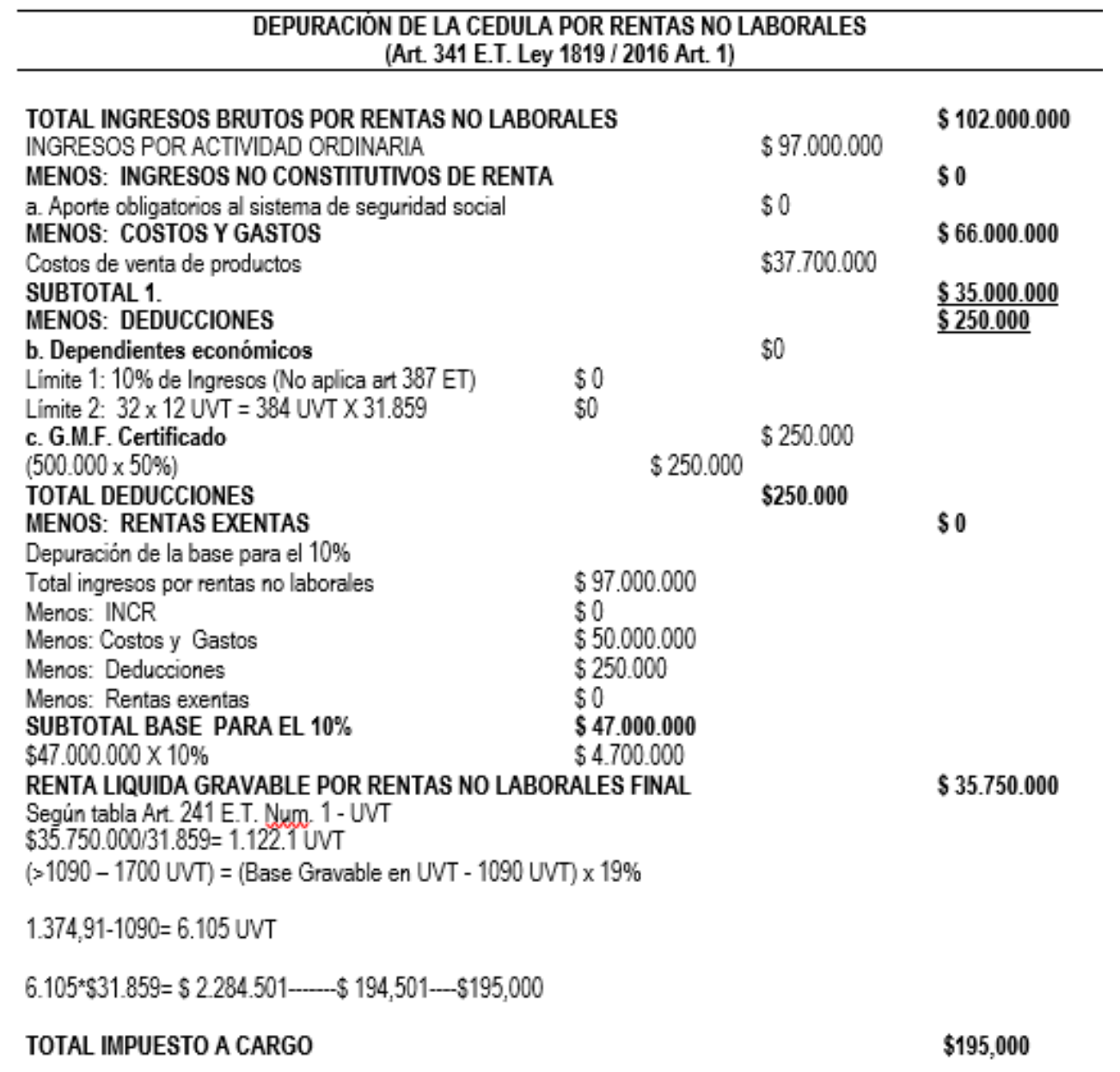


ISSN: 1794-9920 - ISSN Electrónico: 2500-9338

Enero - Julio de 2018

Volumen 18 Número 1, Año 2018 Págs. 6 - 21

Por otro lado, se puede notar que el sistema cedular puede ser diligenciado de forma rápida y concreta, es importante tener en detalle, cuando el comerciante percibe otros ingresos diferentes, si adopta por los sistemas cedula debes reconocerlo en la diferente cedula correspondiente. Ejemplo de ellos los intereses financieros, renta de capital.

\section{CONCLUSIONES:}

Teniendo en cuenta en el desarrollo de los objetivos planteados en el presente estudio que abordó la temática sobre la adopción del Monotributo se permitió percibir que el conocimiento de esta nueva normatividad es de gran importancia para todas las personas, en especial para aquellos comerciantes de menores ingresos, debido a que por medio de este nuevo tributo formalizan sus negocios, obtienen beneficios como lo son acceder a los servicios del sistema general de seguridad social, así como también realizan un aporte a su futura pensión por medio de los BEPS, de igual modo aportan al desarrollo del país al momento de cumplir con sus obligaciones tributarias, por medio de esta normatividad el estado Colombiano pretende captar mayores ingresos de los sectores denominados difíciles de gravar, para así de esta manera la incrementar su plan de acción a través del financiamiento del Sistema General de Seguridad Social, en Salud y en Riesgos Laborales, con el objetivo de mejorar la calidad del sistema de salud nacional, así de esta manera los beneficios podrán ser gozados por la comunidad en general.

Cabe resaltar que la ley 1819 de 2016 trae diversos cambios a nivel general en la legislación tributaria nacional, hablando especialmente sobre la economía podemos decir que la ley busca aumentar el recaudo tributario para su posterior reinversión, pero el desconocimiento e inclusive situaciones concretas como la corrupción generan para los contribuyentes cierta apatía al momento de cumplir con sus obligaciones en materia tributaria, por tal razón es pertinente que exista un acompañamiento y capacitación debido a la novedad de esta alternativa de impuesto para la sociedad, así los comerciantes de menores ingresos puedan acceder a los diferentes beneficios que obtienen al momento de acogerse a dicho tributo, por medio de este de igual manera se busca reducir las cargas formales y sustanciales de los impuestos para así fomentar la formalización de los negocios.

Respecto a la liquidación del impuesto del monotributo en comparación a las renta cedulares, se puedo determinar que los contribuyente que quieran adoptar el nuevo mecanismo, pueden hacer un análisis previo de sus ingresos base de liquidación, calculando la favorabilidad que obtienen en la liquidación de tributo. Si es bien que el

tributo genera beneficios de salud, pensión, también importante analizar los cálculos de pagos sustanciales de los comerciantes, que puede afectar en muchos casos su flujo de efectivo o la disposición para formalizar sus prácticas económica. Dado lo anterior se recomienda que los comerciantes antes de adoptar el mecanismo opcional monotributista establezca en detalles todas las fuentes de ingresos y calcular de acuerdo al tipo de categoría el impuesto a pagar, asimismo compara de acuerdo a la renta no laborales tomando la opción que genere mayor beneficio económico en la liquidación.

\section{REFERENCIAS:}

Arias, M. y R. Pérez. 2015. "Emprendimiento: como comenzar una empresa con éxito". Face: Revista de Ciencias Económicas y Empresariales 14:175-83.

Bustamante, J. P., 2013. "Informalidad y Monotributo", Notas Fiscales, Ministerio de Hacienda y Crédito Público - Dirección General de Política Macroeconómica - Centro de Estudios Fiscales, No. 19

Calero, A. V., 2012. "Políticas para la Formalización de los Trabajadores por Cuenta Propia: el Caso del Monotributo Social", Nota Técnica No. 63 - Empleo e Ingresos - correspondiente al Informe Económico No. 80, Ministerio de Economía y Finanzas Públicas de Argentina.

Castañeda, J.E. (2003). "impuesto sobre las ventas en Colombia”. Bogotá: Editorial Códice Ltda. 
Código Civil. Artículo 74. La existencia legal de toda persona principia al nacer, esto es, al separarse completamente de su madre. Recuperado: http://leyescl.com/codigo_civil/74.htm

Colombia, Decreto 2250 del 2017. Por el cual se adicionan, modifican y sustituyen artículos a los Capítulos 10, 11, 12, 19, 20, 21 y 22 del Título 1 y Capítulos 1 y 7 del Título 4 de la Parte 2 del Libro 1 del Decreto 1625 de 2016, Único Reglamentario en Materia Tributaria, para reglamentar la Parte I de la Ley 1819 de 2016. 29 de Dic, 2017,

Colombia, Decreto 738 de 2017, Por el cual se adiciona el epígrafe de la Parte 5 y un título a la Parte 5 del Libro 1 y se adicionan y modifican literales, incisos y artículos del Título 1 de la Parte 6 del Libro 1 del Decreto 1625 de 2016 Único Reglamentario en Materia Tributaria para reglamentar el Monotributo. 08 May, 2017 Núm. 50227

Colombia, Ley 1819 del 2016. Por medio de la cual se adopta una reforma tributaria estructural, se fortalecen los mecanismos para la lucha contra la evasión y la elusión fiscal, y se dictan otras disposiciones. 29 de Dic, 2016 Núm. 50101.

Colombia, Ley 789 del 2002.Por la cual se dictan normas para apoyar el empleo y ampliar la protección social y se modifican algunos artículos del código sustantivo de trabajo. 27 de Dic, 2002, Diario oficial 45046.

Colombia, Resolución 2015110011305 de 2015, por la cual se prescriben y habilitan unos formularios para el cumplimiento de las obligaciones tributarias en el año 2018. 15 Ener, 2018 Núm. 50478 .

Colombia. Concepto 46572 de 1999, por el cual se establece aclaración del libro fiscal de operaciones diarias. (marzo 25 de 1999).

Colombia. Concepto Dian 15456 de 2006, por cual se aclara disposiciones en relación al régimen simplificado del impuesto a las ventas y libro de contabilidad
Constitución nacional, articulo 334. "La dirección general de la economía estará a cargo del Estado"

Corredor, J.Q. (2009). El impuesto de renta en Colombia 2009. Medellín; Centro interamericano jurídico financiero (CIJUF)

Escobar G.C (2005). Modulo impuesto sobre la renta y complementario, especialización en gestión tributaria. Medellín, Universidad de Antioquia. (documento de docencia).

Estatuto Tributario, Articulo 107. Expensas necesarias son deducibles

Estatuto Tributario, Articulo 241. Tarifa para las personas naturales y extranjeras residentes y asignaciones y donaciones modales.

Estatuto Tributario, Articulo 242. Tarifa especial para dividendos 0 participaciones recibidas por personas naturales residentes.

Estatuto Tributario, Articulo 26. Los ingresos son base de la renta líquida.

Estatuto Tributario, Articulo 340. Ingresos de las rentas no laborales.

Estatuto Tributario, Articulo 341. Renta Líquida Cedular de las Rentas No Laborales

Estatuto Tributario, Articulo 407. Tarifa retención en dividendos y participación

Estatuto Tributario, Articulo 408. Tarifa retención parra rentas de capitales

Estatuto Tributario, Articulo 414-1. Tarifa retención sobre transporte internacional

Estatuto Tributario, Articulo 592. Quienes no están obligados a declarar.

Estatuto Tributario, Articulo 593. Asalariados no obligados a declarar.

Estatuto Tributario, Articulo 905, Sujetos Pasivos

Franco, R. y Martínez, L.F (2010). Contabilidad tributaria un enfoque de armonización. Bogotá: Investigar editores.

González, D. (2006); "Regímenes especiales de tributación para pequeños contribuyentes en América Latina", Banco Interamericano de 
Desarrollo (BID).

Giannini, A. (1957). Instituciones de Derecho Tributario. Traducción española, editorial de Derecho Financiero, Madrid.

Hernández, R., Fernández, C., Baptista, P. (2010) Metodología de la Investigación. 5ta Edición. México, México D.F.: Editorial McGraw Hill.

Hernández, Iván D., Rodrigo Álvarez, Catalina Blanco, y Andrés Carvajal. 2013. "El ascenso de la 'mano invisible': análisis para el surgimiento de un mercado formal de financiación para empresas de base tecnológica (EBT) en Colombia". FACE Facultad de Ciencias Económicas y Empresariales 13:5-32.

Martinez A.G. 2016. "Resumen $\square$ : APPROACHING THE DECISION-MAKING PROCESS IN THE INDUSTRIAL SECTOR SMES CITY DUITAMA, BOYACA , COLOMBIA Abstract $\square$ : SECTOR DA CIDADE DE DUITAMA, BOYACA . COLOMBIA . Resumo $\square ":$ 48-60.

Monsalve, R (2005). Diccionario integrado contable fiscal. Medellín: Centro Interamericano jurídico financiero (CIJUF)

MOUCHARFIECH, David. Propuestas para una reforma tributaria en Venezuela. $X$ Jornadas Venezolanas de Derecho Tributario, Asociación Venezolana de Derecho Tributario, Caracas 2011.

Organización Internacional del Trabajo. (2014). Monotributo en América Latina. Recuperado de http://www.ilo.org/wcmsp5/groups/public/--américas/---ro

lima/documents/publication/wcms_357452.pdf.

Pardo, N., Hoyos, J.l. y Monsalve, R. (2004). Contabilidad y auditoría tributaria. Medellín: Editorial, centro iberoamericano jurídico financiero (CIJUF).

Rincon de Parra, Haydee. 2001. "Calidad, Productividad Y Costos: Analisis De Relaciones Entre Estos Tres Conceptos". Actualidad Contable Faces 4(4):49-61.

Rodríguez, G., Gil, J., García E., y Etxeberría, J. (1996). Análisis de datos cualitativos asistido por ordenador: Aquad y Nudist. Barcelona:PPU.

Rúa, C.A (2007). Conferencia planeación del cierre fiscal 2007. Documento de docencia, universidad del Valle
Sánchez, A., S. Rodríguez y J. Zumaeta, 2000. "Una Propuesta Alternativa al Actual Régimen Especial de Tributación Dirigido a las Pequeñas y Microempresas del Perú", Superintendencia Nacional de Administración Tributaria - SUNAT Perú, XIII Concurso de Monografías CIAT - AEAT - IEF.

Vargas Restrepo Carlos (2017), Contabilidad tributaria. 2 ed. Bogotá: Ecoc Ediciones 2017

Vasco R. (2017). Impuesto sobre las ventas y consumo. CIJUF, Medellín 2017. Pag 23.

Vasco R. (2018), Rentas cedulares para personas naturales y tributación de residentes en el exterior. Editorial CIJUF, Medellín 2018. 
REFLEXIONES EN LA APLICACIÓN DEL MONOTRIBUTO E IMPUESTO SOBRE LA RENTA Y COMPLEMENTARIO EN LOS COMERCIANTES DE MENORES INGRESOS EN COLOMBIA

Deivi David Fuentes Doria - Kevin Díaz Sánchez - Jimmy Ferney Urueña Majul - Johana Elisa Fajardo Pereira

\begin{tabular}{|c|c|c|c|c|c|c|c|c|}
\hline \multicolumn{9}{|c|}{$\begin{array}{c}\text { Anexo N } 1 \\
\text { Grandes empresas del sector cuero, calzado y marroquinería en Colombia }\end{array}$} \\
\hline NIT & Razón social & 2010 & 2011 & 2012 & 2013 & 2014 & 2015 & 2016 \\
\hline 800120681 & Croydon Colombia S.A. & $\mathrm{x}$ & $\mathrm{x}$ & $\mathrm{x}$ & $\mathrm{x}$ & $\mathrm{x}$ & $\mathrm{x}$ & $\mathrm{x}$ \\
\hline 800123954 & Imapieles Ltda. & $x$ & & & & & & \\
\hline 805011316 & $\begin{array}{l}\text { C.I. diseño y moda internacional } \\
\text { S.A.S. }\end{array}$ & $\mathrm{x}$ & $\mathrm{x}$ & $\mathrm{x}$ & $\mathrm{x}$ & $\mathrm{x}$ & & \\
\hline 811015541 & Colombiana de cueros S.A. & $\mathrm{x}$ & $\mathrm{x}$ & $\mathrm{x}$ & $\mathrm{x}$ & $\mathrm{x}$ & & $\mathrm{x}$ \\
\hline 860037037 & Our bag Ltda. & $\mathrm{x}$ & $\mathrm{x}$ & $\mathrm{x}$ & $\mathrm{x}$ & $x$ & $\mathrm{x}$ & $\mathrm{x}$ \\
\hline 860069040 & Industria de calzado jovical S.A. & $x$ & $x$ & $\mathrm{x}$ & $\mathrm{x}$ & $\mathrm{x}$ & $\mathrm{x}$ & $\mathrm{x}$ \\
\hline 890101058 & Curtiembres búfalo S.A. & $\mathrm{x}$ & $x$ & $\mathrm{x}$ & $\mathrm{x}$ & $\mathrm{x}$ & & $\mathrm{x}$ \\
\hline 890104728 & C. I. modapiel S.A. & $\mathrm{x}$ & & $\mathrm{x}$ & & & & \\
\hline 890305571 & La maravilla S.A. & $\mathrm{x}$ & $\mathrm{x}$ & $\mathrm{x}$ & $\mathrm{x}$ & $\mathrm{x}$ & & \\
\hline 890801339 & $\begin{array}{l}\text { Companía manufacturera manisol } \\
\text { S.A. }\end{array}$ & $\mathrm{x}$ & $\mathrm{x}$ & $\mathrm{x}$ & $\mathrm{x}$ & $\mathrm{x}$ & & $\mathrm{x}$ \\
\hline 890900305 & Curtimbres de Itaguí S.A. & $\mathrm{x}$ & $\mathrm{x}$ & $\mathrm{x}$ & $\mathrm{x}$ & $\mathrm{x}$ & & \\
\hline 890913861 & $\begin{array}{l}\text { C.I. uniformes ropa y calzado quin lo } \\
\text { S.A. }\end{array}$ & $\mathrm{x}$ & $\mathrm{x}$ & $\mathrm{x}$ & $\mathrm{x}$ & $\mathrm{x}$ & $\mathrm{x}$ & $\mathrm{x}$ \\
\hline 891408382 & $\begin{array}{l}\text { Americana de curtidos Ltda. y cía } \\
\text { S.C.A. }\end{array}$ & $\mathrm{x}$ & $\mathrm{x}$ & $\mathrm{x}$ & $\mathrm{x}$ & & & $\mathrm{x}$ \\
\hline 800085026 & Curtipieles S.A.S. & & $x$ & & $x$ & $x$ & $x$ & \\
\hline 860009034 & Stanton S.A.S. & & $x$ & $\mathrm{x}$ & $\mathrm{x}$ & $\mathrm{x}$ & $\mathrm{x}$ & $\mathrm{x}$ \\
\hline 890504493 & Mussi zapatos S.A.S. & & $\mathrm{x}$ & $\mathrm{x}$ & $\mathrm{x}$ & $x$ & $\mathrm{x}$ & $\mathrm{x}$ \\
\hline 800191700 & Cueros vélez S.A.S. & & & & $\mathrm{x}$ & $x$ & $\mathrm{x}$ & $\mathrm{x}$ \\
\hline 805014351 & Plasticaucho Colombia S.A. & & & & $\mathrm{x}$ & & & $\mathrm{x}$ \\
\hline 890904603 & Fábrica de calzado setenta S.A. & & & & $\mathrm{x}$ & & & $\mathrm{x}$ \\
\hline 800116749 & Extractora monterrey S.A. & & & & & $\mathrm{x}$ & $\mathrm{x}$ & \\
\hline 800223739 & Atunes y enlatados del caribe S.A. & & & & & $\mathrm{x}$ & & \\
\hline 802005075 & Aceites S.A. & & & & & $x$ & $x$ & \\
\hline 804017043 & Extractora central S.A. & & & & & $x$ & $x$ & \\
\hline 830034702 & Marketing de Colombia S.A. C.I. & & & & & $\mathrm{x}$ & $\mathrm{x}$ & \\
\hline 900056129 & Extractora la paz S.A. & & & & & $\mathrm{x}$ & $\mathrm{x}$ & \\
\hline 900062992 & Evacol S.A.S. & & & & & $\mathrm{x}$ & $\mathrm{x}$ & $\mathrm{x}$ \\
\hline 900114122 & Extractora maria la baja S.A. & & & & & $\mathrm{x}$ & & \\
\hline 800092777 & Grupo nova S.A. & & & & & & $\mathrm{x}$ & \\
\hline 890924742 & Fábrica de calzado kondor Ltda. & & & & & & $\mathrm{x}$ & $\mathrm{x}$ \\
\hline 800196632 & Artesa S.A.S. & & & & & & & $\mathrm{x}$ \\
\hline 860508043 & Proalpet S.A. C.I. & & & & & & & $\mathrm{x}$ \\
\hline 800020706 & Nalsani S.A.S. & & & & & & & $\mathrm{x}$ \\
\hline 860047483 & Industrias alimenticias aretema S.A. & & & & & & & $\mathrm{x}$ \\
\hline 891400726 & Alpaca S.A.S. & & & & & & & $\mathrm{x}$ \\
\hline & Total & 13 & 14 & 14 & 17 & 22 & 16 & 20 \\
\hline
\end{tabular}

